\title{
Factors Determining Decay-Index Distribution and Contribution of a Central Solenoid on a Spherical Tokamak
}

\author{
Kichiro SHINYA \\ AITEL Corporation, Yokohama 235-8523, Japan
}

(Received 23 January 2006 / Accepted 30 May 2006)

\begin{abstract}
Factors that dominate achievement of the decay-index (or N-index) distribution required for highly elongated divertor plasmas with large triangularity are studied. The outermost coil is regarded as a leading candidate, but can supply only a part of the equilibrium value. Current in Central Solenoid with the same direction as the outermost coil current is found to fill the difference of the decay-index distribution on the mid-plane between the equilibrium magnetic field and the outermost coil field.
\end{abstract}

(c) 2006 The Japan Society of Plasma Science and Nuclear Fusion Research

Keywords: spherical tokamak, elongated plasma, triangularity, equilibrium field, decay-index, poloidal field coil, central solenoid

DOI: $10.1585 /$ pfr. 1.036

Highly elongated plasma with an appropriate triangularity is favorable for achieving good plasma performances and attaining divertor configurations with reasonable divertor coil currents. Usually, elongated plasmas are associated with a negative decay-index of the equilibrium magnetic field at the plasma center. Furthermore, the inboard plasma surface needs to be selectively pushed near mid-plane by the magnetic field to attain high triangularity plasma. Since the direction of the magnetic line of force of such a pushing field is opposite to that of the vertical field, the pushing field weakens the vertical field near the inboard plasma surface. This causes the decay-index to be more negative at the inboard plasma surface, so the precise investigation of the decay-index distribution is inevitable for such highly shaped plasma. The decay-index at any major radius is defined as follows:

$$
N=-\frac{R}{B_{Z}} \frac{\partial B_{Z}}{\partial R} .
$$

The right-hand cylindrical coordinate system, $(R, \varphi, Z)$, is used. $B_{Z}$ is an axial component of the magnetic field or vertical field. The plasma current is positive throughout this paper. A magnetic field with a negative decay-index will presumably be produced by the outermost coil (a pair of upper and lower coils) current, because a coil with a small angle of poloidal opening, as shown in Fig. 1, can effectively produce highly elongated plasmas [1]. The minimum angle is limited by the size of the largest horizontal port.

In this paper, the factors that dominate the decayindex distribution required for a highly elongated $(\kappa=2.5)$ divertor plasma with a large triangularity $(\delta=0.7)$ are evaluated using the plasma parameters and the poloidal field coil (PFC) configuration of the Plasma Bound-

author'se-mail: shinya@pic.keihin.toshiba.co.jp

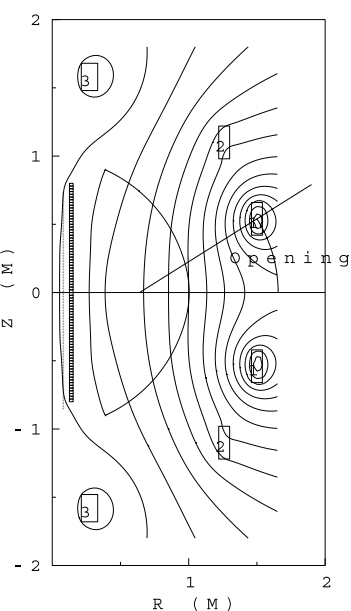

(a)

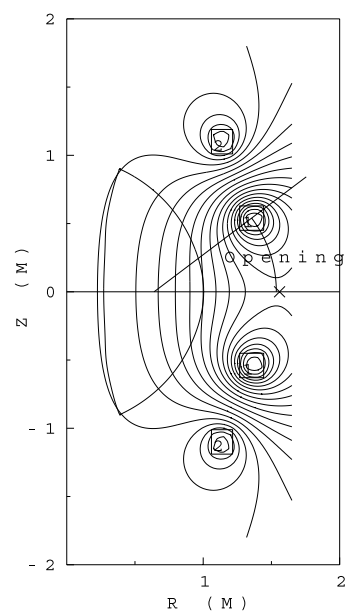

(c)

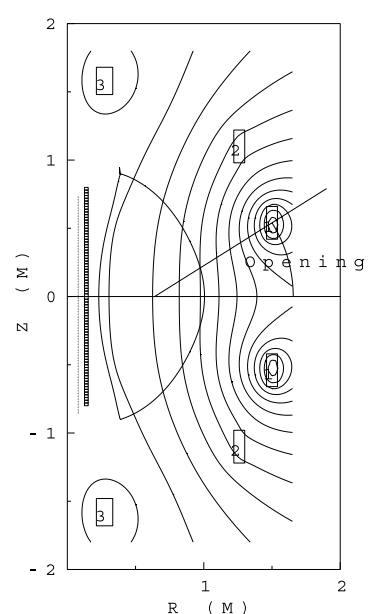

(b)
Fig. 1 Magnetic field configuration of the double null divertor plasma equilibrium for (a) the QUEST CS and (b) the Uniform CS. (c) Magnetic field profile produced by PF1 and $\mathrm{PF} 2$ excitation for the reduced $\mathrm{PF} 1$ and $\mathrm{PF} 2$ radii. $\mathrm{PF} 2$ and PF1 currents are $70 \mathrm{kAT}$ and $-100 \mathrm{kAT}$, respectively $(\mathrm{PF} 2 / \mathrm{PF} 1=-0.7)$. 
Table 1 Coil locations and equilibrium currents for Reference and Reduced sets.

\begin{tabular}{|c|c|c|c|c|c|c|c|}
\hline & \multicolumn{4}{|c|}{ Reference set } & \multicolumn{3}{|c|}{ Reduced set } \\
\hline Coil \# & $\mathrm{R}(\mathrm{m}$ & $\mathrm{Z}(\mathrm{m})$ & $\mathrm{I}(\mathrm{kAT})$ & I (kAT) & $\mathrm{R}(\mathrm{m})$ & $\mathrm{Z}(\mathrm{m})$ & $\mathrm{I}(\mathrm{kAT})$ \\
\hline PF1 & 1.5 & 0.54 & -99.1 & -99.2 & 1.36 & 0.54 & -98.3 \\
\hline PF2 & 1.2 & 1.10 & -18.6 & -10.0 & 1.13 & 1.10 & -8.9 \\
\hline PF3 & $0.2^{2}$ & 1.50 & 213.2 & 125.9 & 0.27 & 1.50 & 176.0 \\
\hline CS & 0.1 & 2.75 & -566.0 & -729.4 & 0.14 & 2.75 & -573.3 \\
\hline$\overline{C S}$ type & & & QUEST & Uniform & & & QUEST \\
\hline
\end{tabular}

ary Dynamics Experimental Device at Kyushu University (QUEST hereafter). In the QUEST design, ports of $0.45 \mathrm{~m}$ height $\times 0.7 \mathrm{~m}$ width are equipped, and thus the height of the outermost coil is $0.54 \mathrm{~m}$ making an angle of $32.1^{\circ}$.

First, dependence of the decay-index at the plasma center (geometrical center of the plasma cross section, i.e., $0.64 \mathrm{~m}$ ) upon the outermost coil radius was studied, maintaining its height with $0.54 \mathrm{~m}$. Despite the relatively small poloidal angle of the outermost coil opening, the coils provide only a small part of the decay-index required for the QUEST equilibrium and the assistances of the other coils are necessary. Next, the contribution of the currents in the neighboring coils was investigated. If the current directions of the two coils are opposite, the magnetic lines of force are introduced inward through the aperture between the outermost coil and the neighboring coil, which supplies curvature of the magnetic line of force with negative decay-index. While adequate positive currents of neighboring coils can attain the decay-index required for the QUEST equilibrium at the plasma center, the distribution of the decay-index on the mid-plane differs greatly from that of the equilibrium magnetic field. The negative current in an appropriately configured central solenoid (CS) is found to fill the difference of the decay-index distributions. It should be noted that the outermost coil currents are negative throughout the study.

Figure 1 (a) shows the equilibrium magnetic field for the double null divertor plasma. The value of the decayindex at the plasma center is -0.21 . The plasma current is $300 \mathrm{kA}$ that requires a $-0.07 \mathrm{~T}$ vertical field at the plasma center. The major and the minor radii are $0.64 \mathrm{~m}$ and $0.36 \mathrm{~m}$, respectively. The plasma shape and coil locations are also shown in the figure. Note that the CS is $1.6 \mathrm{~m}$ long and has twice the current density at the central part of $0.8 \mathrm{~m}$. The equilibrium field has larger curvature at the inboard surface, decreasing in the radial direction. This is common in the equilibrium with large triangularity. Coil locations and currents are summarized in Table 1 as the QUEST CS type. PF1 corresponds to the outermost coil. PF3 is often called a divertor coil.

It is worthwhile to examine the benefit of the QUEST $\mathrm{CS}$ for plasma shaping. Figure 1 (b) shows the equilibrium magnetic field configuration and the resultant plasma shape for the Uniform CS. A clear difference is seen near the inboard plasma surface. The inboard plasma surface of the Uniform CS inflates near the mid-plane, which causes deterioration of the elongation and the triangularity. Equilibrium coil currents of the Uniform CS are also shown in Table 1 as the Uniform CS in the Reference set. There exist remarkable differences of the PF3 and CS currents between the QUEST CS and the Uniform CS. Note that the PF2 current is negative for both CS types.

Reduction of the PF1 radius might decrease the electric power of the PF1 coil. The reduction, on the contrary, increases an angle of the poloidal opening, which in turn induces decay-index toward the positive value. Dependences of the decay-index and the vertical field on the PF1 radius are shown in Fig. 2 (a). Only the upper and lower PF1 coils are excited. Currents are $-100 \mathrm{kAT}$ which is a rounded value in both the QUEST CS and the Uniform CS, as is shown in Table 1. The absolute value of the decay-index decreases with decreasing PF1 radius, and it becomes positive for a radius less than $1.358 \mathrm{~m}$. At a much larger PF1 radius, it becomes saturated and then its absolute value gradually decreases to zero. On the contrary, magnetic field strength decreases linearly with coil radius. Even in the saturation region, the value of the decay-index is less than half of that required. The PF1 radius of the present design seems to be reasonable from the viewpoint of trade-off between decay-index and magnetic field strength.

Figure 2 (b) depicts the supplementary effect of the positive currents in the neighboring coils (PF2). The PF1 current is $-100 \mathrm{kAT}$ and the radius is $1.358 \mathrm{~m}$ at which the decay-index by PF1 decreases to zero. The PF2 radius is decreased proportionally to the PF1 radius from the present design. The decay-index on the plasma center recovers the equilibrium value at $I_{2} / I_{1}=-0.7$ where $I_{2}$ and $I_{1}$ are the PF2 and PF1 currents, respectively. The magnetic field configuration for $I_{2} / I_{1}=-0.7$ is shown in Fig. 1 (c). It is clear that the curvature distribution along the major radius is different from that of the equilibrium. Plasmas with smaller triangularities will be produced by this magnetic field.

The reduced set in Table 1 corresponds to poloidal coil locations with reduced $\mathrm{PF} 1$ and $\mathrm{PF} 2$ radii such that the decay index at the plasma center produced only by PF1 current disappears. The PF2 radius is reduced proportionally. 


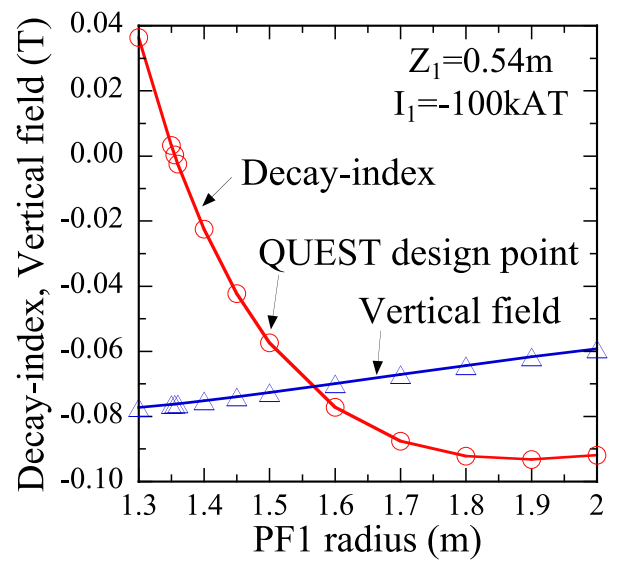

(a)

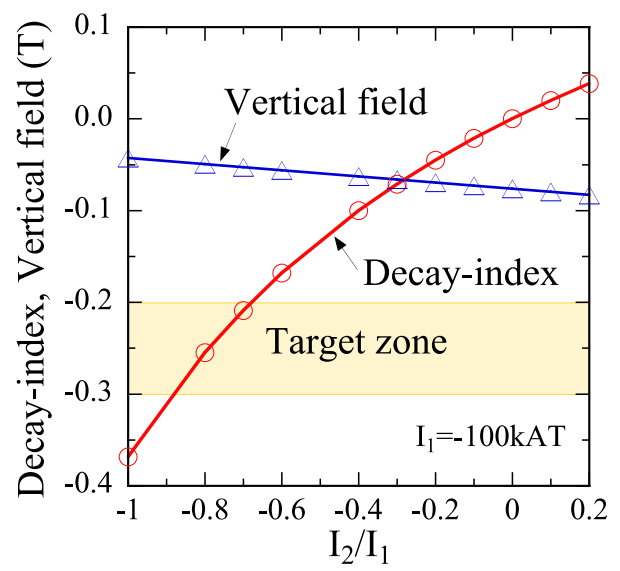

(b)

Fig. 2 (a) Dependence of the decay-index and vertical magnetic field at the plasma center on the PF1 radius when only PF1 is excited. (b) Dependence of the decay-index and vertical magnetic field at plasma center on the ratio between the PF2 and PF1 currents for the reduced PF1 and $\mathrm{PF} 2$ radii. $\mathrm{PF} 1$ and $\mathrm{PF} 2$ are simultaneously excited. The PF1 current is kept at $-100 \mathrm{kAT}$. Target zone corresponds to the decay-index of the QUEST equilibrium.

Plasma shape is basically the same as that of the equilibrium with the reference $P F 1$ and PF2 locations and QUEST type CS. A slight difference was observed in the position of the outboard plasma surface. PF1 and CS currents are essentially the same as the Reference QUEST design. About 50 and 20 percent differences were observed in PF2 and PF3 currents, respectively. Negative PF2 current, which weakens the decay-index produced by PF1, indicates that the supplement by PF2 to the decay-index cannot be anticipated in the QUEST equilibrium. These results suggest that the CS current may be important for the plasma shaping.

Decay-index distributions for the equilibrium with different CS type are compared in Fig. 3. The QUEST_CS_Eq and the Uniform_CS_Eq corresponds to the equilibrium with QUEST type CS and Uniform CS, respectively. Uniform CS cannot provide a sufficient decay-

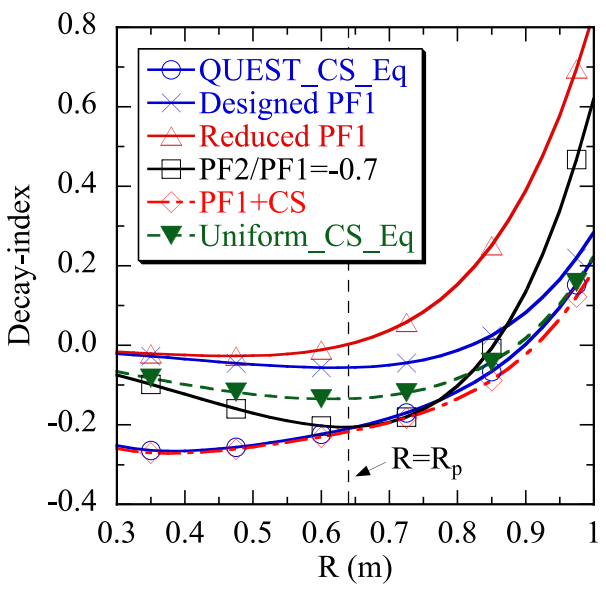

Fig. 3 Decay-index distributions of the equilibrium magnetic field with different CS type are denoted by _EQ at the end. Other cases are the distributions for the magnetic fields produced by selective excitation of the PF coils and/or CS.

index at the inside half of the plasma cross section. Distributions for the magnetic field produced by selective excitation of PF coils and/or CS are also shown in the figure. In these cases, only a part of the coils is excited to study an effect of the individual coil(s). The coil current listed in Table 1 is used except in the case of PF2/PF1 $=-0.7$. The decay-index only with PF1 excitation (Designed PF1, Reduced PF1) shifts in the positive direction from the equilibrium value in the whole plasma region. $\mathrm{PF} 2$ excitation with $70 \% \mathrm{PF} 1$ current in the reduced $\mathrm{PF}$ set $(\mathrm{PF} 2 / \mathrm{PF} 1=-0.7)$, i.e., $\mathrm{PF} 2=70 \mathrm{kAT}$ and $\mathrm{PF} 1=-100 \mathrm{kAT}$, recovers the decay-index only at the plasma center. It should be emphasized that the QUEST type CS contributes to achieving the decay-index distribution of the equilibrium completely (shown as PF1 $+\mathrm{CS}$ ). In this case, the coil currents of QUEST CS listed in Table 1 are used for both PF1 and CS. Curvature of the stray field from CS in the plasma region is opposite to that by PF1 with a small poloidal opening.

The opposite curvature by CS helps the curvature by PF1 to achieve the equilibrium distribution because the decay-index can be separated into contributions from CS and the other coils to the axial component of the magnetic field.

$$
N=-\frac{R}{B} \frac{\partial B}{\partial R}=-\frac{R}{B_{\mathrm{PF}}+B_{\mathrm{CS}}} \frac{\partial}{\partial R}\left(B_{\mathrm{PF}}+B_{\mathrm{CS}}\right) .
$$

Here the suffixes CS and PF mean contributions from the $\mathrm{CS}$ and the other poloidal coils, respectively. Usually $\left|B_{\mathrm{CS}}\right|$ is much smaller than $\left|B_{\mathrm{PF}}\right|$, so the above equation is approximated by

$$
N \simeq\left(1-\frac{B_{\mathrm{CS}}}{B_{\mathrm{PF}}}\right) N_{\mathrm{PF}}+\frac{B_{\mathrm{CS}}}{B_{\mathrm{PF}}} N_{\mathrm{CS}} .
$$

If the CS current is negative, both $B_{\mathrm{CS}}$ and $N_{\mathrm{CS}}$ are positive, 
resulting in an increase in the absolute value of the decayindex. Substituting the values for the equilibrium magnetic field shown in Fig. 1 (a), i.e., $B_{\mathrm{PF}}=-7.6 \times 10^{-2} \mathrm{~T}$, $B_{\mathrm{CS}}=5.9 \times 10^{-3} T, N_{\mathrm{PF}}=-6.1 \times 10^{-2}$ and $N_{\mathrm{CS}}=1.72$, we have $N=-0.199$. The value agrees with the equilibrium value with only a $5 \%$ error. A shorter CS can produce a larger $N_{\mathrm{CS}}$, In QUEST, the central part of the CS has twice the line density of the current, therefore produces a large decay-index effectively. $\left|B_{\mathrm{CS}}\right|$ increases with decreasing major radius while $\left|B_{\mathrm{PF}}\right|$ decreases, hence the decay-index at the inboard plasma surface becomes further negative which is the case for the equilibrium with large triangularity. Effects of PF2 and PF3 (divertor coil) are supposed to shape the magnetic field apart from the mid-plane. The fine shape of the plasma surface and the magnetic field configuration near the x-point, for example, will be controlled by PF2 and PF3.
In conclusion, (1) the outermost coil cannot produce a sufficient decay-index of the equilibrium field required for the highly elongated divertor plasma with large triangularity; (2) A neighboring coil with opposite current direction to the outermost coil current supplements the decay-index only at the plasma center. The distribution along the major radius is not coincident with the equilibrium field. The neighboring coil current might be positive or negative for the QUEST equilibrium depending on the plasma parameters and the shape; (3) An appropriately configured CS with larger current in the central part helps PF1 by filling the difference in the decay-index distribution from the equilibrium magnetic field on the mid-plane, if the CS current flows in the same direction as the PF1 current.

[1] K. Shinya, J. Plasma Fusion Res. 80, 949 (2004) [in Japanese]. 